\title{
Bionomics of Anopheles latens in Kapit, Sarawak, Malaysian Borneo in relation to the transmission of zoonotic simian malaria parasite Plasmodium knowlesi Cheong H Tan ${ }^{1,2}$, Indra Vythilingam*1, Asmad Matusop ${ }^{3}$, Seng T Chan ${ }^{1}$ and Balbir Singh ${ }^{2}$
}

Address: ${ }^{1}$ Infectious Diseases Research Centre, Institute for Medical Research Jalan Pahang, 50588 Kuala Lumpur, Malaysia, ${ }^{2}$ Malaria Research Centre, University Malaysia Sarawak., Kuching, Sarawak, Malaysia and ${ }^{3}$ Sarawak Department of Health, Kuching, Sarawak, Malaysia

Email: Cheong H Tan - anopheles1972@yahoo.com; Indra Vythilingam* - indra@imr.gov.my;

Asmad Matusop - asmad.matusop@health.gov.my; Seng T Chan - sengthim@yahoo.com; Balbir Singh - bsingh@fmhs.unimas.my

* Corresponding author

Published: 31 March 2008

Malaria Journal 2008, 7:52 doi:10.1186/1475-2875-7-52
Received: II February 2008

Accepted: 31 March 2008

This article is available from: http://www.malariajournal.com/content/7/l/52

(c) 2008 Tan et al; licensee BioMed Central Ltd.

This is an Open Access article distributed under the terms of the Creative Commons Attribution License (http://creativecommons.org/licenses/by/2.0), which permits unrestricted use, distribution, and reproduction in any medium, provided the original work is properly cited.

\begin{abstract}
Background: A large focus of human infections with Plasmodium knowlesi, a simian parasite naturally found in long-tailed and pig-tailed macaques was discovered in the Kapit Division of Sarawak, Malaysian Borneo. A study was initiated to identify the vectors of malaria, to elucidate where transmission is taking place and to understand the bionomics of the vectors in Kapit.

Methods: Three different ecological sites in the forest, farm and longhouse in the Kapit district were selected for the study. Mosquitoes were collected by human landing collection at all sites and at the forest also by monkey-baited-traps situated on three different levels. All mosquitoes were identified and salivary glands and midguts of anopheline mosquitoes were dissected to determine the presence of malaria parasites.

Results and Discussions: Over an II-month period, a total of 2,504 Anopheles mosquitoes comprising 12 species were caught; I,035 at the farm, 774 at the forest and 425 at the longhouse. Anopheles latens (62.3\%) and Anopheles watsonii (30.6\%) were the predominant species caught in the forested ecotypes, while in the farm Anopheles donaldi (49.9\%) and An. latens (35.6\%) predominated. In the long house, An. latens (29.6\%) and An. donaldi (22.8\%) were the major Anopheline species. However, An. latens was the only mosquito positive for sporozoites and it was found to be attracted to both human and monkey hosts. In monkey-baited net traps, it preferred to bite monkeys at the canopy level than at ground level. An. latens was found biting early as 18.00 hours.

Conclusion: Anopheles latens is the main vector for P. knowlesi malaria parasites in the Kapit District of Sarawak, Malaysian Borneo. The study underscores the relationship between ecology, abundance and bionomics of anopheline fauna. The simio-anthropophagic and acrodendrophilic behaviour of An. latens makes it an efficient vector for the transmission of $P$. knowlesi parasites to both human and monkey hosts.
\end{abstract}




\section{Background}

Malaria parasites in Peninsular Malaysian monkeys were first reported in 1908 [1], but only gained prominence in the 1960's after the accidental discovery [2] that Plasmodium cynomolgi could be transmitted to humans via mosquito bites in the laboratory. This stimulated interest at a time when the Malaria Eradication Programme was initiated by the World Health Organization [3] and it was important to determine if malaria was a zoonosis. Therefore, extensive studies were carried out in Peninsular Malaysia to determine the distribution, prevalence and species of malaria parasites in monkeys and apes and the natural vectors of monkey malaria parasites [4-7]. Instead of uncovering human cynomolgi malaria infections, Plasmodium knowlesi was the first simian malaria parasite found to be infecting humans in nature. The first case was reported in 1965 from the state of Pahang [8], Peninsular Malaysia, followed by a second case five years later acquired from Johore, Peninsular Malaysia [9]. It was postulated that $P$. knowlesi could be transmitted from monkeys to man and laboratory studies proved that it was possible [10]. However, a large scale study that was initiated in Pahang to investigate whether malaria was a zoonosis, by a group of American and local researchers based at the Institute for Medical Research in Kuala Lumpur, Peninsular Malaysia, concluded that simian malaria in humans was an extremely rare event $[11,12]$. This was based on their studies in which they collected blood samples from more than 1,100 local residents, pooled the samples and injected them into rhesus monkeys and none of the monkeys contracted malaria. However, in 2004 a large focus of human $P$. knowlesi infection was reported in the Kapit Division of Sarawak [13]. In that study $71.6 \%$ (101/141) of human malaria cases at Kapit Hospital which had been identified by microscopy as single Plasmodium malariae infections were actually $P$. knowlesi and other non-P. malariae species by nested polymerase chain reaction (PCR) assays. Plasmodium knowlesi is naturally found in long-tailed macaques (Macaca fascicularis), pigtailed macaques (Macaca nemestrina) [14] and banded leaf monkeys (Presbytis malalophos) $[15,16]$. Since transmission of this zoonotic parasite to humans is occurring in the Kapit Division of Sarawak, Malaysian Borneo, it is important to identify the vectors so that appropriate measures can be planned and initiated to control the spread of simian malaria in humans.

Numerous studies on vectors of human malaria have been carried out in Sarawak [17-20] but none of these have been undertaken in the Kapit District of Sarawak. Several anopheline species incriminated or suspected to transmit monkey malaria parasites in Peninsular Malaysia, such as Anopheles latens, Anopheles balabacensis are also present in Sarawak [21]. Hence, the vectorial status of the anopheline species present in the Kapit Division needs to be ascertained in order to determine if they are competent vectors of simian malaria parasites. Furthermore, the epidemiological data of $P$. knowlesi infection in humans revealed that infections occur primarily in adults and no clustering of cases occurred within communities that live in communal longhouses, which suggests transmission of $P$. knowlesi to humans occurred away from the vicinity of the longhouses [13].

Thus the objectives of this study were to determine the vectors of $P$. knowlesi and other simian malaria parasites in the Kapit Division of Sarawak; and to study the dynamics of these vectors in different ecological sites in order to elucidate the most likely place where transmission was taking place. Preliminary results of this study were reported where An. latens was incriminated as the vector for $P$. knowlesi [22]. Here detailed results of this eleven-month study on vectors of malaria and their bionomics in the Kapit district of Sarawak, Malaysian Borneo is presented.

\section{Methods \\ Study sites}

The study was carried out in Kapit district from June 2005 to April 2006. The district is part of Kapit division located in the central part of Sarawak, Malaysian Borneo. Kapit division is bordered by Kalimantan, Indonesia in the south and east, Miri and Bintulu divisions in the north and Sibu division in the west. The Kapit district is a hilly area covered by tropical rain forests. Three different ecological sites were selected based on human cases of $P$. knowlesi and or presence of long-tailed macaques (M. fascicularis). The first site was a longhouse (residence of the indigenous people) in Lubok Loh Yong situated $16 \mathrm{~km}$ from the town center and is close to the river and surrounded by trees and shrubs. One of the residents of the longhouse had been admitted to Kapit Hospital with knowlesi malaria in 2004. The second site was a farm in Ulu Sungai Yong on land cleared of original forest and now supporting fruit trees and secondary vegetation. There is a small hut in the farm and a stream is running through the farm. There is only one house situated just outside the farm. Pig-tailed macaques (M. nemestrina) are often sighted in this area and one of the farmers had previously been admitted to Kapit Hospital with knowlesi malaria. The distance between the longhouse and farm is $5 \mathrm{~km}$. The third site was a forest $4.5 \mathrm{~km}$ away from Kapit town center and is 1,200 meters above sea level. The forest is only accessible by four wheel drive vehicle but during the rainy season it is only accessible by foot. Long-tailed macaques were often seen in this area, which is also approximately $1 \mathrm{~km}$ from the house of a malaria patient admitted to Kapit Hospital in 2001 with knowlesi malaria. 


\section{Mosquito collections}

All night mosquito collections, using the bare-leg catch method [23] were performed during the eleven-month study period. In each area, three nights of collections were carried out every month. In the longhouse both indoor and outdoor collections were carried out by two men each while in the farm and forest fringe only outdoor collections were performed by three men working in two shifts. The catches were performed from 18.00 to 06.00 hours.

\section{Monkey-baited-trap}

In the forest, a comparison was made between the number of mosquitoes attracted to human bait on the ground and the numbers attracted to monkey bait at ground level, on platforms at three meters and six meters on the forest canopy. In the first two months only one monkey was placed in each cage but in subsequent months there were two monkeys per cage. The cages with the monkeys were placed on the platform inside a mosquito net measuring $190 \mathrm{~cm} \times 180 \mathrm{~cm} \times 150 \mathrm{~cm}$ with an opening of about $40 \mathrm{~cm}$ on either ends. The method used was similar to that used by Wharton [24]. Platforms were constructed among the branches of a tree. The traps were operated from 18.00 to 06.00 hours and were searched at two hourly intervals. A collector, upon entering the net, closed the opening and collected all resting mosquitoes with the use of a suction tube.

\section{Mosquito identification and dissection}

All mosquitoes were identified morphologically in the field laboratory. The keys of Reid [21] were used for the identification of Anopheles mosquitoes. Generally most Anopheles mosquitoes were dissected to extract ovaries for parity determination and the midguts and salivary glands were examined for oocysts and sporozoites respectively. The remaining pair of the salivary glands of all parous anopheline mosquitoes were placed in an individual 1.5 $\mathrm{ml}$ microcentrifuge tubes (Axygen, USA) containing 95\% alcohol and were labeled accordingly. In addition, all positive midguts were preserved using the same method. The preserved salivary glands and midguts were used for molecular studies [22].

\section{DNA extraction and PCR}

Ethanol used to preserve the salivary glands and midguts of malaria positive mosquitoes were allowed to evaporate completely. To accelerate the evaporation, all $1.5 \mathrm{ml}$ tubes containing the preserved specimens were incubated in a dry bath (Thermomixer, Eppendorf, Germany) set at $70^{\circ} \mathrm{C}$. Genomic DNA of the dried salivary glands was extracted using DNeasy ${ }^{\circledast}$ tissue Kit (Qiagen, Germany) and performed according to the manufacturer's protocol. The eluted DNA was kept at $4^{\circ} \mathrm{C}$ until required. A nested PCR assay [13] based on the Plasmodium DNA sequence of the small subunit ribosomal RNA (SSUrRNA) genes was used to detect and identify the species of malaria parasites found in Anopheles mosquitoes caught in Kapit.

\section{Ethical clearance}

This project was approved by the Medical Research \& Ethics Committee Ministry of Health Malaysia (KKM/JEPP/ 02(110). All volunteers who carried out mosquito collections were provided with antimalarial prophylaxis.

\section{Results}

Species composition and spatial distribution of Anopheles species collected in different ecological sites

A total of 2,504 Anopheles mosquitoes belonging to 12 species were caught biting humans and monkeys (Table 1). Of these, 10 species were obtained biting man while two (Anopheles pujutensis, Anopheles macarthuri) were caught exclusively biting monkeys. Anopheles latens comprised $42.9 \%$ of the total collection. Besides An. latens, the two predominant species were Anopheles donaldi in the farm and Anopheles watsonii in the forest.

There were observed differences in species composition and abundance between different ecological sites. Anopheles latens (62.3\%) and An. watsonii (30.6\%) were the predominant species caught in the forested ecotype, while in the farming area An. donaldi (49.9\%) and An. latens (35.6\%) were predominant. In the long-house, An. latens (29.7\%), An. donaldi (22.8\%) and Anopheles vanus (21.9\%) were found to be the major anopheline species caught, albeit the numbers of mosquitoes caught indoors were less as compared to the outdoor collection. Anopheles kokhani, a species new to science [25], was also observed in all three sites. The rest of the species were found in varying numbers depending on where they were collected.

Table I: Mosquitoes collected from three different ecological sites in Kapit, Sarawak from June 2005-April 2006.

\begin{tabular}{lllllll}
\hline Anopheles species & Farm & \multicolumn{2}{l}{ Long House } & Forest & Total (\%) \\
\cline { 3 - 7 } & & In & Out & BLC & MBT \\
\hline An. barbirostris & 5 & 0 & 3 & 0 & 0 & $8(0.3)$ \\
An. donaldi & 651 & 35 & 62 & 2 & 1 & $751(30)$ \\
An. introlatus & 3 & 0 & 1 & 0 & 0 & $4(0.2)$ \\
An. latens & 465 & 37 & 89 & 273 & 209 & $1073(42.8)$ \\
An. macarthuri & 0 & 0 & 0 & 0 & 8 & $8(0.3)$ \\
An. maculatus & 6 & 0 & 1 & 11 & 2 & $20(0.8)$ \\
An. pujutensis & 0 & 0 & 0 & 0 & 9 & $9(0.4)$ \\
An. roperi & 0 & 1 & 2 & 0 & 0 & $3(0.1)$ \\
An. tesselatus & 9 & 15 & 38 & 0 & 0 & $62(24.8)$ \\
An. vanus & 84 & 25 & 68 & 2 & 3 & $182(7.3)$ \\
An. watsonii & 1 & 1 & 1 & 157 & 80 & $240(9.6)$ \\
An. kokhani & 81 & 19 & 27 & 15 & 2 & $144(5.8)$ \\
\hline Total & 1305 & 133 & 292 & 460 & 314 & 2504 \\
\hline
\end{tabular}




\section{Changes in landing rate over time}

The biting peak of An. latens in the farm was in June and from October to December 2005 and in February 2006, while in the forest there was an increase in biting rate in October and November 2005 and in April 2006 (Figure 1 ). However, in the longhouse, the peak was observed to be in October 2005. Studies in the past have shown that the amount of rainfall affects the anopheline biting density $[26,27]$. During the study period there was no meteorological station in Kapit and the nearest meteorological station was more than $100 \mathrm{~km}$ away in the Division of Sibu. Consequently, it was not possible to determine the effect of rainfall on the monthly densities of mosquitoes in this study.

\section{Biting cycles}

In the forest, An. latens comes to bite as early as 18.00 hours in the evening but the peak biting time is between 19.00 and 20.00 followed by a sharp decline thereafter as shown in Figure 2. While in the farm it starts to bite from 18.00 hours and continues to bite throughout the night until dawn (06.00 hours) and the peak biting time is between 01.00 to 02.00 hours. In the longhouse, An. latens showed a peak outdoor biting time between $23.00 \mathrm{~h}$ to $02.00 \mathrm{~h}$ while a peak biting time indoor between midnight and $02.00 \mathrm{~h}$ was observed.

\section{Monkey-baited trap}

A total of 312 Anopheles mosquitoes were caught in the monkey-baited trap. Of these, An. latens was the predominant species followed by An. watsonii. The results of mosquitoes obtained from the monkey-baited traps are summarized in Table 2. Anopheles latens preferred to bite at six meters rather than three meters and ground level. The ratio of ground:3 m:6 m was as follows, 1:10.3:18.4. Most were found entering the trap between 18.00 to 21.00 hours. Based on results from this study, the An. latens biting ratio of monkey to human was $1: 1.3$.

\section{Parous rate, daily survival rate, life expectancy and vectorial capacity of Anopheles latens}

The parous rates of An. latens and the confidence interval is shown in Table 3. Overall, more than $59 \%$ of the An. latens caught in Kapit were parous, however a significant difference was observed between the parous rates of $A n$. latens caught in different ecological sites. The parity rate observed in An. latens caught at the farm was similar to those collected in the longhouse $(65.8 \%)$. However, the parous rate of An. latens caught at the forest was observed to be significantly lower than those caught at the farm $\left(\mathrm{X}^{2}\right.$ $=13.27, \mathrm{P}<0.05)$ and the longhouse $\left(\mathrm{X}^{2}=5.67, \mathrm{P}<0.05\right)$.

The daily survival rate [28], life expectancy [29] and vectorial capacity [30] of An. latens are shown in Table 3. Overall life expectancy of $A n$. latens was highest in the farm and

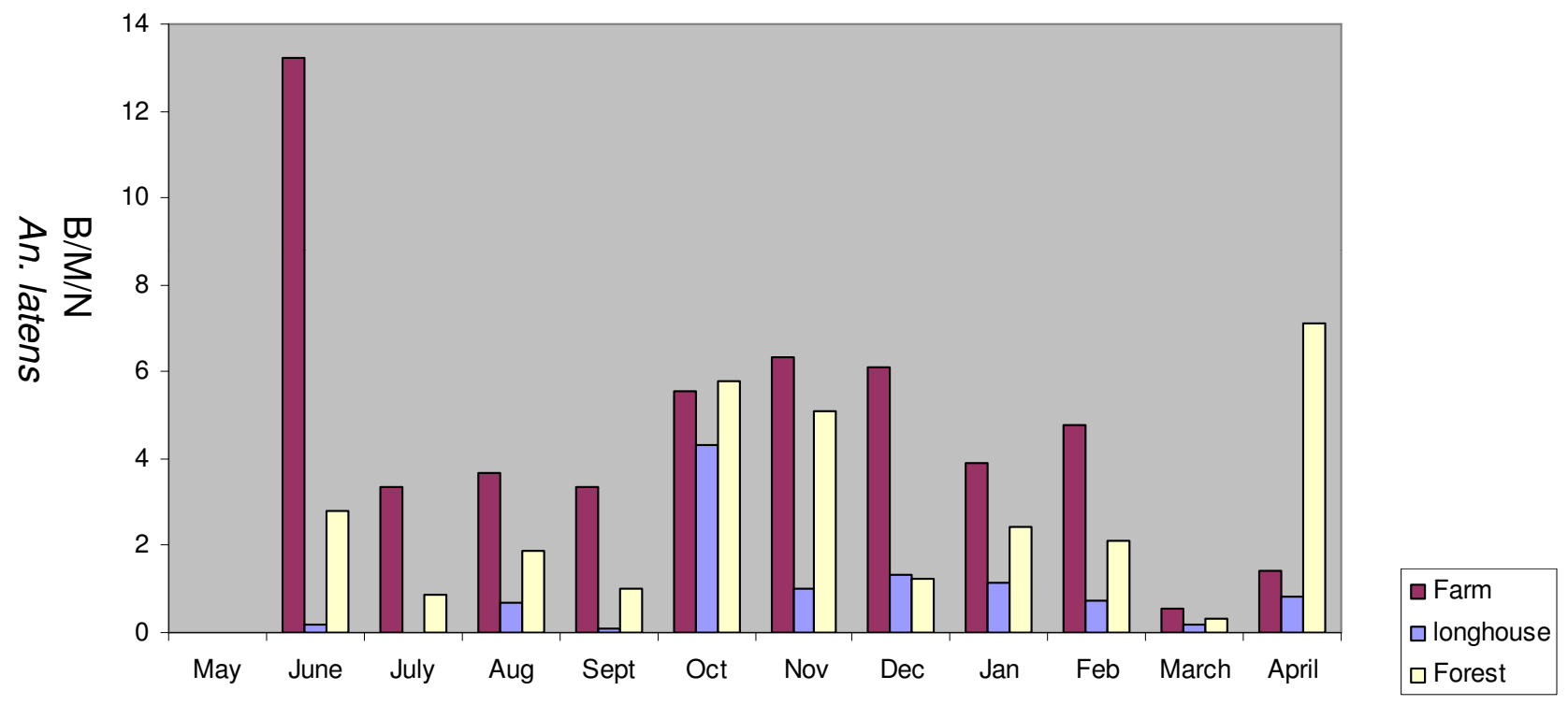

Months

Figure I

Vector density of Anopheles latens at the study sites. 


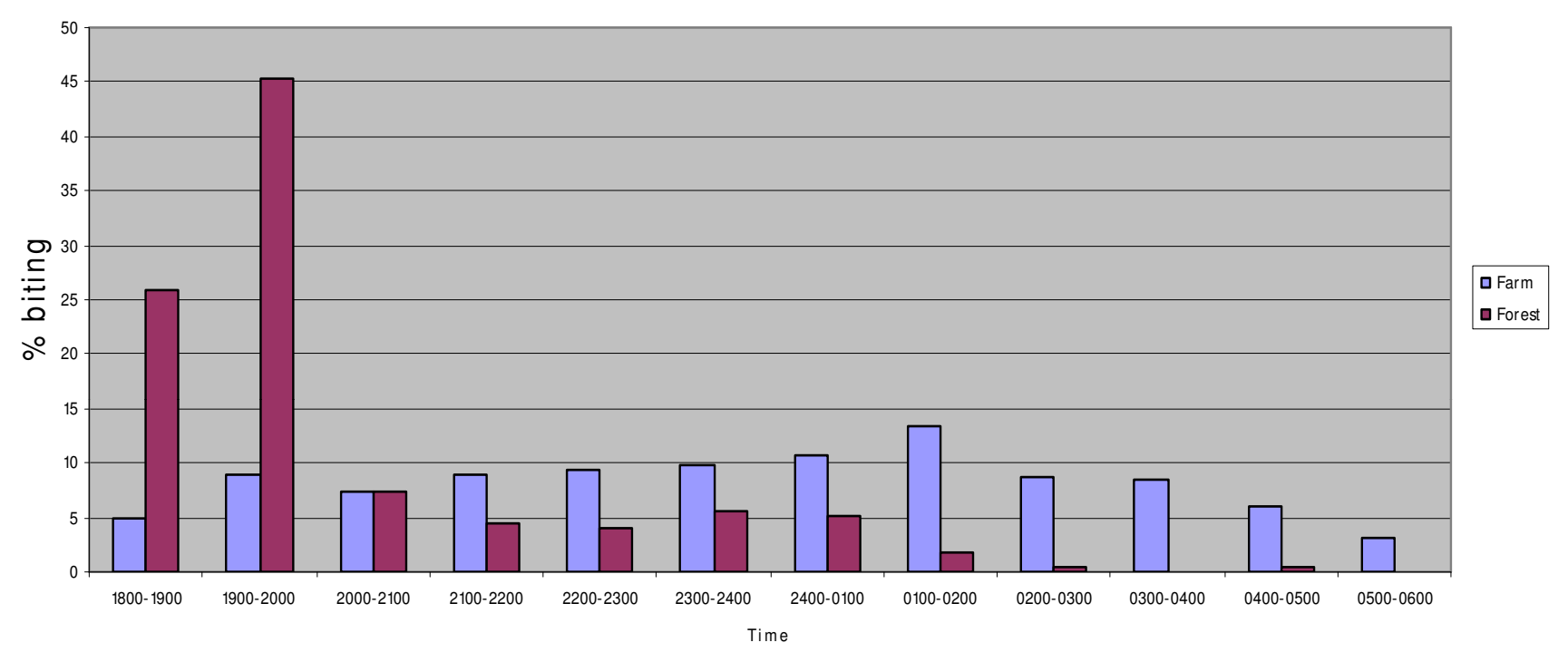

Figure 2

Biting times of Anopheles latens in farm and forest.

longhouse and followed by those found in the forest. In the farm and long-house $25 \%$ of An. latens would be expected to live the 10 days necessary for the P. knowles $i$ sporozoites to be formed. Those surviving the 10 days would have a further life expectancy of 7.2 days. For this calculation results of mosquitoes caught outdoors were pooled together with the results of those caught inside the long-house. The vectorial capacity of the An. latens was found to be highest in the farm, and lowest in the forest.

\section{Sporozoite rate and annual entomological inoculation rates}

A total of nine An. latens were found to harbour the sporozoite stage of malaria parasites and all were of simian origin. Of these, six were caught in the forest, and the rest in the farm area. Two of the infective An. latens, caught in the forest, were found to be positive for P. knowlesi [22], including one that was caught in the monkey-baited net trap. In addition, two An. latens caught attracted to humans in the farm, were also observed to harbour the sporozoite stage of $P$. knowlesi [22]. The remaining five

Table 2: Anopheles latens obtained at the monkey-baited-traps

\begin{tabular}{lllllll}
\hline & 21 hours & 23 hours & 0100 & 0300 & 0500 & Total (\%) \\
\hline Ground & 1 & 2 & 3 & 0 & 1 & $7(3.3)$ \\
3 Meters & 41 & 12 & 9 & 4 & 7 & $73(34.9)$ \\
6 Meters & 47 & 25 & 32 & 6 & 19 & $129(61.7)$ \\
\hline Total & 89 & 38 & 44 & 10 & 27 & 209 \\
\hline
\end{tabular}

infective An. latens were found to carry other species of simian malaria parasites. The overall sporozoite rates in both the farm and the forest were $0.7 \%$ and $1.4 \%$, respectively (Table 4). The computed annual entomological inoculation rates (EIR) were 11.98 and 14.10 in the farm and in the forest, respectively. However, the annual EIR for P. knowlesi was 7.8 and 4.6 in the farm and forest respectively and this in not statistically significant $\left(\mathrm{X}^{2}=\right.$ $0.22 \mathrm{P}>0.05$ ).

There were five An. latens, seven An. watsonii and one An. donald $i$ that were positive for the oocyst stage (Table 4). In addition, three of the infective An. latens, that were caught in the forests, also had oocysts in their midgut (Table 4). None of the mosquitoes collected from the longhouse had malaria parasites

\section{Discussion}

In the 1960's, it was suggested that mosquitoes of the Anopheles leucosphyrus group, may provide a link between humans and monkeys and that if simian malaria is transmitted to man in nature, it is likely to be in areas where these mosquitoes are common [31]. This has been confirmed by the current study where An. latens has been incriminated as the vector of $P$. knowlesi in nature in the Kapit division [22] where a large focus of naturally acquired $P$. knowlesi infection in humans was reported [13]. Aside from being the most predominant anopheline mosquitoes caught biting humans in Kapit, An. latens was observed to be attracted to both human and monkey hosts. In the monkey-baited net traps, it was also observed 
Table 3: Parous rate, probability of survival, probability of transmission of malaria parasite, life expectancy and vectorial capacity of An. latens

\begin{tabular}{lccc}
\hline & Farm & Forest & Longhouse \\
\hline Parous rate $(95 \% \mathrm{Cl})$ & $65.8(61.2-70.1)$ & $53.7(48.9-58.3)$ & $65.8(57.0-73.9)$ \\
Probability of daily survival -P & 0.87 & 0.81 & 0.87 \\
$\mathrm{P}^{10}(\%)$ & 25 & 13 & 25 \\
Life expectancy P10/-log $\mathrm{P}$ (days) & 7.2 & 4.7 & 7.2 \\
Vectorial capacity & 2.86 & 0.60 & 0.85
\end{tabular}

$p^{10}$ percentage of population expected to live long enough to become infective with an extrinsic cycle of 10 days based on P. knowlesi extrinsic incubation period [32].

that a significant number of An. latens were collected at the higher levels compared to those caught at ground level showing an acrodendrophilic behaviour. This is important as the natural monkey host is arboreal in nature. In order for this malaria parasite to be maintained in nature and for transmission to man to occur, the vector needs to be highly simio-anthropophagic in nature. If the vector for $P$. knowlesi is highly simiophagic, as in the case of Anopheles hackeri in Peninsular Malaysia [4], transmission in the natural hosts may be maintained, but transmission to humans will be rare [32].

The fact that An. latens is attracted to monkeys in the canopy and humans on the ground indicates that both humans and monkeys could be exposed to infection from each other. Data from this study area indicate that the zoonotic parasite, $P$. knowlesi, is being transmitted to both humans and macaques by An. latens. Thus, in Kapit, humans get the infection when their forest associated activities, such as farming, logging, or hunting exposes them to An. latens. Throughout the course of this study infected mosquitoes were not obtained from the longhouse and the number of anophelines caught there were also small.

Like all members of the Leucosphyrus group, An. latens is mainly a forest breeding mosquito associated with dense jungle and forest fringes $[21,33]$. In the current study, nearly $90 \%$ of the total An. latens were collected at either the farm $(40 \%)$ or the forest $(50 \%)$ and were consistent with earlier studies conducted in other areas of Sarawak.
In addition, only 126 An. latens (10\%) were collected in the longhouse, and of these $71 \%$ were collected outdoors. Entomological studies conducted in other areas in Sarawak $[18,19]$ showed that An. latens were commonly found in farming zones that were located at the forest fringe rather than in villages, and the density of An. latens decreases in relation to distance away from the jungle.

It is known that multiple factors determine the prevalence of malaria and one of these is the intensity of malaria transmission which is defined as the rate at which people get inoculated with malaria parasites from mosquitoes [34]. In the current study, only An. latens were found to be infective, and the average number of infectious bites by these mosquitoes in both the farm and the forest during the entire duration of the study was 11.98 and 14.1 per year respectively, which is higher compared to the rates in many Asian countries [34]. When considering the risk of acquiring $P$. knowlesi in Kapit, the intensity of transmission has been shown to be comparable for both ecotypes, hence the risk of acquiring $P$. knowlesi in either the forest or the farm is the same. In Sarawak, the forests and their surrounding areas are recognized focal points for malaria transmission whenever An. latens is present in large numbers [20].

Although An. latens has been incriminated as the vector of $P$. knowlesi in Kapit, the role of other anopheline species as a possible vector for $P$. knowlesi in Kapit needs further assessment. This is especially true for An. pujutensis, An. introlatus, and An. macarthuri, all of which were caught in

Table 4: Sporozoite and Oocyst rate of positive mosquitoes in study sites

\begin{tabular}{lllllll}
\hline Mosquito Species & \multicolumn{2}{l}{ Number dissected } & \multicolumn{2}{c}{ Sporozoite Rate $(95 \% \mathrm{Cl})$} & Oocyst Rate $(95 \% \mathrm{Cl})$ \\
\cline { 2 - 7 } & Farm & Forest & Farm & Forest & Farm & Forest \\
\hline An. latens & 435 & 438 & $0.69(0.18-2.18)$ & $1.37^{*}(0.56-3.11)$ & $0.46(0.08-1.84)$ & $1.37(0.56-3.11)$ \\
An. watsonii & 1 & 224 & 0 & 0 & 0 & $3.13(1.38-6.60)$ \\
An. donaldi & 641 & 1 & 0 & 0 & $0.16(0.01-1.00)$ & 0 \\
\hline
\end{tabular}

* One of the infective An. latens was caught in the monkey baited net trap 
small numbers and were not dissected. They have been reported to be simiophagic and have either been suspected or been incriminated as vectors of simian malaria parasites in other localities in Peninsular Malaysia $[32,35,36]$. To determine if they are vectors of $P$. knowlesi, intensive entomological surveys have to be carried out in many parts of the forest and in other parts of the Kapit Division. With the advancement of molecular techniques it is possible to identify the sporozoites to species level and thus vectorial status can be determined.

Anopheles watsonii, a forest species, was caught in large numbers, but only in the forest, where it was found to be attracted to both human and monkey hosts. Previously, a small numbers of this species were also obtained in hill forest in Peninsular Malaysia at night with human and monkey- baited traps [35]. They obtained more mosquitoes in the canopy than at ground level, but all were negative by dissection. Thus earlier workers did not consider An. watsonii as a vector of human malaria. In the current study, oocysts were found by dissection and the numbers in each gut ranged from 7-90. However, none were positive for sporozoites by dissection. Since they are attracted to humans and non human primates, further studies should be conducted on this species before any definitive conclusions can be inferred about their vectorial status.

With large tracts of forest being cut down, non-human primates are coming close to the human environment. Human cases of $P$. knowlesi have been reported not only in Sarawak[13], but also in Thailand [37], Manymar [38] and in Peninsular Malaysia [8,9,39]. Thus control strategies for malaria in Southeast Asia may have to change to include this zoonotic aspect of the transmission.

\section{Conclusion}

This study has shown that An. latens, previously incriminated as the main vector for $P$. knowlesi in Kapit District of Sarawak, Malaysian Borneo has the highest vectorial capacity among all Anopheline species caught in the area. The abundance and composition of anopheline species was found to be dependent on the ecotype studied, with An. latens being predominantly found in the forest and farm. The simio-anthropophagic and acrodendrophilic behaviour of An. latens has been established. These evidence-based findings will be useful for the planning of control strategies for malaria vectors.

\section{Competing interests}

The author(s) declare that they have no competing interests.

\section{Authors' contributions}

IV and BS conceived the study. IV, CHT, BS were responsible for preparation of manuscript. IV, CHT, STC and AM were responsible for field collection, supervision, identification and processing of mosquitoes. BS obtained permits to collect and undertake research on biological specimens from Sarawak Biodiversity Council and permits to trap and keep monkeys in captivity from Sarawak Forestry Department. CHT conducted molecular work. All authors have read and approved the manuscript.

\section{Acknowledgements}

The support staff of the respective departments is greatly appreciated. We gratefully acknowledge Bruce Harrison and Kevin Palmer for reviewing the manuscript and providing constructive comments, the Sarawak Biodiversity Council for permission to collect mosquitoes for the study and to use monkey-baited traps for the study (Permit No: SBC-RP-008I (i)-BS), the Forestry Department of Sarawak, for permission to trap and keep long-tailed macaques in captivity (Permit No. NPW.907.4.2 (I)-98), David Lee for suggestion of possible sites for mosquito collection, and the Director, Institute for Medical Research, Kuala Lumpur, for permission to publish. This project was funded by the Ministry of Health Malaysia, grant MRG2005-4 and trapping of macaques was supported by a fundamental research grant from Universiti Malaysia Sarawak (0I [82].422/2004 [59]).

\section{References}

I. Daniels CW: Animal parasites in man and some of the lower animals in Malaya. Stud Inst Med Res FMS 1908, 3:1-13.

2. Eyles DE, Coatney GR, Getz ME: Vivax-type malaria parasite of macaques transmissible to man. Science 1960, 132:1812-1813.

3. Sandosham AA: Recent researches on malaria at the Institute for Medical Research, Kuala Lumpur. Med J Malaya 1967, 22: $145-160$.

4. Wharton RH, Eyles DE: Anopheles hackeri, a vector of Plasmodium knowlesi in Malaya. Science 1961, 134:279-280.

5. Wharton RH, Eyles DE, Moorhouse DE: Anopheles leucosphyrus identified as a vector of monkey malaria in Malaya. Science 1962, 137:758.

6. Eyles DE, Warren McW, Guinn E, Wharton RH, Ramachandran CP: Identification of Anopheles balabacensis introlatus as a vector of monkey malaria in Malaya. Bull World Health Organ 1963, 28:134-I35.

7. Cheong WH, Warren McW, Omar AH, Mahadevan S: Anopheles balabacensis balabacensis identified as a vector of simian malaria in Malaysia. Science 1965, 150:314-1315.

8. Chin W, Contacos PG, Coatney RG, Kimbal HR: A naturally acquired quotidian-type malaria in man transferable to monkeys. Science 1965, 149:865.

9. Yap LF, Cadigan FC, Coatney GR: A presumptive case of naturally occurring Plasmodium knowlesi malaria in man in Malaysia. Trans R Soc Trop Med Hyg 1971, 65:839-840.

10. Garnham PCC: Malaria parasites and other Haemosporidia Blackwell Scientific Publications, Oxford; 1966.

II. Sandosham AA, Eyles DE, Yap LF: Plasmodium cynomolgi bastianellii from a Malayan monkey and its similarity to aberrant P. vivax in man. Med J Malaya 1962, 17:78-79.

12. Warren McW, Cheong WH, fredericks HK, Coatney GR: Cycles of jungle malaria in West Malaysia. Am J Trop Med Hyg 1970, 19:8I-10I.

13. Singh B, Lee KS, Matusop A, Radhakrishnan A, Shamsul SSG, CoxSingh J, Thomas A, Conway DJ: A large focus of naturally acquired Plasmodium knowlesi infections in human beings. Lancet 2004, 363:1017-1024.

14. Eyles DE, Laing ABG, Dobrovolny CG: The malaria parasites of the pig-tailed macaque, Macaca nemstrina (Linnaeus), in Malaya. Ind J Malar 1962, 16:285-298.

15. Eyles DE, Laing ABG, Warren M, Sandoshan AA: Malaria parasites of the Malayan leaf monkeys of the genus Presbytis. Med J Malaya 1962, 17:85-86.

16. Eyles DE: The species of simian malaria: taxonomy, morphology, lifecycle and geographical distribution of the monkey species. J Parasit 1963, 49:866-867. 
17. Colless DH: The Anopheles leucosphyrus group. Trans R Entomol Soc Lond 1956, 108:37-II6.

18. Chang MS, Doraisingam P, Hardin S, Nagum N: Malaria and filariasis transmission in a village/forest setting in Baram district, Sarawak, Malaysia. J Trop Med Hyg 1995, 98: I92-198.

19. Chang MS, Hii J, Buttner P, Mansoor F: Changes in abundance and behaviour of vector mosquitoes induced by land use during development of an oil palm plantation in Sarawak. Trans $R$ Soc Trop Med Hyg 1 997, 9 I:382-386.

20. Chang MS, Asmad M, Fam KS: Differences in Anopheles composition and malaria transmission in the village settlements and cultivated farming zone in Sarawak, Malaysia. Southeast Asian J Trop Med Pub Health 1999, 30:454-459.

21. Reid JA: Anopheline mosquitoes of Malaya and Borneo. Stud Inst Med Res Malaysia 1968, 3 I:520.

22. Vythilingam I, Tan CH, Asmad M, Chan ST, Lee KS, Singh B: Natural transmission of Plasmodium knowlesi to humans by Anopheles latens in Sarawak, Malaysia. Trans R Soc Med Hyg 2006, I00(I I): 1087-1088

23. Vythilingam I, Foo LC, Chiang GL, Chan ST, Eng KL, Mahadevan S, Mak JW, Inder Singh K: The impact of permethrin impregnated bednets on the malaria vector Anopheles maculatus (Diptera:Culicidae) in aboriginal villages of Pos Betau Pahang, Malaysia. Southeast Asian J Trop Med Pub Health 1995, 26(2):354-358.

24. Wharton RH, Eyles DE, Warren McW: The development of methods for trapping the vectors of monkey malaria. Ann Trop Med Parasit 1963, 57:32-46.

25. Vythilingam I, Jeffery J, Harbach RE, Chan ST, Tan CH, Asmad M: Anopheles (Cellia) kokhani N. sp (Diptera: Culicidae) from Kapit, Sarawak, East Malaysia. Proc Entomol Soc Wash 2007, 100:463-468.

26. Trung HD, Van Bortel W, Sochantha T, Keokkenchanh K, Quang NT, Cong LD, Coosemans M: Malaria transmission and major vectors in different geographical areas of Southeast Asia. Trop Med Int Health 2004, 9:230-237.

27. Vythilingam I, Chan ST, Shanmugaratnam C, Tanrung H, Chooi KH: The impact of development and malaria control activities on its vectors in the Kinabatangan area of Sabah, East Malaysia. Acta Trop 2005, 96:24-30.

28. Davidson G: Estimation of survival rate of anopheline mosquitoes in nature. Nature 1954, 174:792-793.

29. Garret-Jones C, Grab B: The assessment of insecticidal impact on the malaria mosquito's vectorial capacity from data on the proportion of parous females. Bull World Health Organ 1964, 3I:7I-86.

30. Garret-Jones C, Shidrawi GR: Malaria vectorial capacity of a population of Anopheles gambiae, an exercise in epidemiological entomology. Bull World Health Organ 1969, 40:531-545.

31. Coatney GR, Collins WE, Contacos PG: The primate malarias US Government Printing Office. Washington; 197I.

32. Warren $\mathrm{McW}$, Wharton $\mathrm{RH}$ : The vectors of simian malaria: identity, biology and geographical distribution. J Parasit 1963, 49:892-904.

33. Sallum MAM, Peyton EL, Wilkerson RC: Six new species of the Anopheles leucosphyrus group, reinterpretation of An. elegans and vector implications. Med Vet Entomol 2005, 19:158-199.

34. Olumese P: Epidemiology and surveillance: changing the global picture of malaria - myth or reality? Acta Trop 2005, 95:265-269.

35. Wharton RH, Eyles DE, Warren McW, Cheong WH: Studies to determine the vectors of monkey malaria in Malaya. Ann Trop Med Parasit 1964, 58:56-77.

36. Reid JA, Weitz B: Anopheline mosquitoes as vectors of animal malaria in Malaya. Ann Trop Med Parasit 196I, 55:180-186.

37. Jongwutiwes S, Putaporntip C, Iwasaki T, Sata T, Kanbara H: Naturally acquired Plasmodium knowlesi malaria in human, Thailand. Emerg Infect Dis 2004, 10:22 I I-22 I3.

38. Zhu HM, Li J, Zheng $\mathrm{H}$ : Human natural infection of Plasmodium knowlesi. Zhongguno Ji Sheng Chong Xue Yu ji Sheng Chong Bing Za Zhi 2006, 24(I):70-7I.

39. Cox-Singh J, Davis TME, Lee KS, Shamsul SSG, Matusop A, Ratnam S, Rahman HA, Conway DJ, Singh B: Plasmodium knowlesi malaria in humans is widely distributed and potentially life-threatening. Clinical Infect Dis 2007, 46(2): I65-I7I.
Publish with Bio Med Central and every scientist can read your work free of charge

"BioMed Central will be the most significant development for disseminating the results of biomedical research in our lifetime. "

Sir Paul Nurse, Cancer Research UK

Your research papers will be:

- available free of charge to the entire biomedical community

- peer reviewed and published immediately upon acceptance

- cited in PubMed and archived on PubMed Central

- yours - you keep the copyright 